\title{
edoc
}

Institutional Repository of the University of Basel

University Library

Schoenbeinstrasse 18-20

CH-4056 Basel, Switzerland

http://edoc.unibas.ch/

Year: 2015

\section{Automated analysis of spine dynamics on live CA1 pyramidal cells}

Blumer, Clemens and Vivien, Cyprien and Genoud, Christel and Perez-Alvarez, Alberto and Wiegert, J. Simon and Vetter, Thomas and Oertner, Thomas G.

Posted at edoc, University of Basel

Official URL: http://edoc.unibas.ch/dok/A6328773

Originally published as:

Blumer, Clemens and Vivien, Cyprien and Genoud, Christel and Perez-Alvarez, Alberto and Wiegert, J. Simon and Vetter, Thomas and Oertner, Thomas G.. (2015) Automated analysis of spine dynamics on live CA1 pyramidal cells. Medical image analysis, Vol. 19, H. 1. S. 87-97.

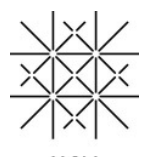




\section{Automated analysis of spine dynamics on live CA1 pyramidal cells}

Clemens Blumer ${ }^{1}$, Cyprien Vivien ${ }^{2}$, Christel Genoud ${ }^{2}$, Alberto Perez-Alvarez ${ }^{3}$, J. Simon Wiegert ${ }^{3}$, Thomas Vetter ${ }^{1 \alpha^{*}}$, Thomas G. Oertner ${ }^{3 \alpha^{*}}$

${ }^{1}$ Department of Mathematics and Computer Science, University of Basel, Bernoullistrasse $16, \mathrm{CH}$ 4056 Basel, Switzerland

${ }^{2}$ Friedrich Miescher Institute for Biomedical Research, Maulbeerstrasse 66, CH-4058 Basel, Switzerland

${ }^{3}$ Center for Molecular Neurobiology Hamburg, Falkenried 94, D-20251 Hamburg, Germany

\& These authors contributed equally

*corresponding authors:

Thomas G. Oertner

Center for Molecular Neurobiology Hamburg

Falkenried 94

20251 Hamburg

Germany

Email: thomas.oertner@zmnh.uni-hamburg.de

Phone: +49 40741058228

Thomas Vetter

Department of Mathematics and Computer Science

University of Basel

Bernoullistrasse 16

4056 Basel

Switzerland

Email: thomas.vetter@unibas.ch

Phone: +416126705 56

Figures: 6 color figures

Supplemental material: 3 supplemental figures

DOI: http://dx.doi.org/10.1016/j.media.2014.09.004

Preprint submitted to Medical Image Analysis, September 2014 


\title{
Highlights
}

- Spine tracking over time using statistical models and probability maps

- Correlative two-photon/electron microscopy datasets used for benchmarking

- Analysis of spine orientation and detection precision in organotypic slice culture

- Application 1: Automatic identification of synaptically connected spines

- Application 2: Automatic analysis of organelle motility in spines

\begin{abstract}
Dendritic spines may be tiny in volume, but are of major importance for neuroscience. They are the main receivers for excitatory synaptic connections, and their constant changes in number and in shape reflect the dynamic connectivity of the brain. Two-photon microscopy allows following the fate of individual spines in brain slice preparations and in live animals. The diffraction-limited and non-isotropic resolution of this technique, however, makes detection of such tiny structures rather challenging, especially along the optical axis (z-direction). Here we present a novel spine detection algorithm based on a statistical dendrite intensity model and a corresponding spine probability model. To quantify the fidelity of spine detection, we generated correlative datasets: Following twophoton imaging of live pyramidal cell dendrites, we used serial block-face scanning electron microscopy (SBEM) to reconstruct dendritic ultrastructure in 3D. Statistical models were trained on synthetic fluorescence images generated from SBEM datasets via point spread function (PSF) convolution. After the training period, we tested automatic spine detection on real two-photon datasets and compared the result to ground truth (correlative SBEM data). The performance of our algorithm allowed tracking changes in spine volume automatically over several hours. Using a second fluorescent protein targeted to the endoplasmic reticulum, we could analyze the motion of this organelle inside individual spines. Furthermore, we show that it is possible to distinguish activated spines from non-stimulated neighbors by detection of fluorescently labeled presynaptic vesicle clusters. These examples illustrate how automatic segmentation in $5 D(x, y, z, t, \lambda)$ allows us to investigate brain dynamics at the level of individual synaptic connections.
\end{abstract}

Keywords: dendritic spines, spine detection, CA1 pyramidal cells, 2-photon microscopy, hippocampus, endoplasmic reticulum, image segmentation, statistical models 


\section{Introduction}

Imaging live neurons is a powerful technique to investigate the functionality of the brain. Twophoton microscopy (2PM), which uses infrared light to locally excite fluorescence, is especially suited to image fine neuronal structures deeply embedded in intact tissue. To study the dynamics of excitatory synaptic connections between neurons, dendritic spines are often used as a proxy: the size of a dendritic spine is correlated with the strength of the synapse impinging on it (Matsuzaki et al., 2001), and the density of spines on the dendrite is altered in many mental disorders (Nimchinsky et al., 2002). The tiny volume of dendritic spines is below the resolution limit of light microscopy and therefore not easy to measure or to track over time. While fast scanning systems and motorized microscopes have made it possible to generate large amounts of high-resolution fluorescence images in relatively short time, detailed analysis and quantification of these large datasets poses a severe bottleneck. Manual analysis, placing regions of interest (ROI) on individual structures, is time consuming, and the results may vary with the skill and ability of the human analyst. In addition, this type of analysis is often done on maximum intensity projections (MIP), ignoring any information in the axial ( $z$ ) direction. Automatic detection, segmentation and evaluation of dendritic spines in 3D fluorescence datasets would be very valuable, especially for the analysis of time series data (4D). In the past, several approaches for automatic spine detection were presented, driven mostly by skeleton and backbone reconstruction. In these approaches, spines are detected as short side branches from the dendritic backbone (Cheng et al., 2007; Zhang et al., 2007; Janoos et al., 2009; Yuan et al., 2009). Other skeletonizing approaches detect additionally the tip of spines and use them to segment spines via grassfire transform or similar (Koh et al., 2002; Rodriguez et al., 2007; Xu et al., 2006; Zhou et al. 2008). The performance of all automatic detection programs strongly depends on resolution and contrast of the input images. As different datasets were used for benchmarking, reported values for precision and recall cannot be directly compared between studies.

A related problem, the automatic tracing of axonal and dendritic branching patterns, has benefitted from direct performance comparison in form of an organized competition (DIADEM challenge; Brown et al., 2011; , Gilette et al., 2011). Successful segmentation of dendritic spines, however, was not a criterion in this competition. From a methodological point of view it is interesting that one of hardest problems in automatic backbone reconstruction, the correct merging of separated branches, has recently been tackled successfully by machine learning (Gala et al., 2014). Learning of locally invariant features in 3D can also be applied to the spine detection problem (Fehr, 2009). Objective evaluation of spine detection performance is difficult. Usually, fluorescence images annotated by human experts are used as ground truth, and the quality of automatic detection is then benchmarked against the manually annotated fluorescence dataset. The resolution of light microscopes including confocal and two-photon microscopes, however, is limited by diffraction. Especially in axial direction, fine spatial features are efficiently filtered out in the process of imaging, and small spines protruding in axial direction are easily missed by automatic detection and by human experts, too.

Here, we rely on the superior resolution of electron microscopy to generate ground truth and training datasets in a machine learning approach to spine detection. Our approach uses knowledge about the typical shape and size of spines on a particular type of neuron and knowledge about the spatial resolution of a particular microscope to detect spines in noisy images. Statistical shape models have proven to be a powerful and unbiased approach to face recognition and related problems (e.g. Active Shape Models (ASM) (Cootes et al., 1995), 3D Morphable Model (Blanz and 
Vetter 1999). To generate statistical models of dendritic spines, it is essential to use training data in which spines can be identified and annotated unambiguously. As diffraction-limited light microscopy does not provide sufficient spatial resolution, we performed serial block-face scanning electron microscopy (SBEM, (Denk and Horstmann, 2004; Briggman and Denk, 2006)) on neurobiotin-filled neurons of interest (CA1 pyramidal cells). From high-contrast SBEM data, we generated dendritic surface models. Reconstructed dendritic volume was convolved with the point-spread-function (PSF) of our two-photon microscope to generate synthetic fluorescence images (SFIs). SFIs were resampled orthogonal to the dendritic backbone to generate 2D slices at regular intervals. After a registration procedure to compensate for the non-isotropic resolution of SFIs, two statistical models were generated by PCA: One of the characteristic cross-section of a spiny dendrite, and a second one containing information about the presence or absence of a spine in that cross-section.

After training of the statistical models, we tested the performance of spine detection on data that were not part of the training set. For this benchmarking procedure, we produced correlative datasets of spiny dendrites by 2-photon live cell microscopy, subsequent tissue fixation, and SBEM. Using correlative two-photon/EM data overcomes a fundamental problem of expert-labeled fluorescence data: no expert can detect spines that, after filtering by the point-spread-function (PSF) of the microscope, leave no recognizable trace in the light microscopy data. The superior resolution of SBEM data revealed an interesting, non-random orientation of spines in organotypic culture and allowed to us to benchmark our detection software in an objective fashion. In addition to the prediction maps generated by approximation with the statistical models, we analyzed intensity changes along the backbone to provide a second criterion for the presence of dendritic spines.

Once we were satisfied with the performance of our program, we addressed two biologically relevant questions as application examples. First, we tried to automatically detect spines that were synaptically connected to fluorescently labeled axons from other neurons. To evaluate the performance of the automatic analysis, we compared the results to spine calcium transients triggered by optogenetic stimulation of the labeled presynaptic axons (Wiegert and Oertner, 2013). Spines with functional synaptic connections were successfully identified. As a second example, we analyzed the dynamics of endoplasmic reticulum (ER) which moves in and out of dendritic spines, potentially altering synaptic properties (Holbro et al., 2009). As multiple color channels $(\lambda)$ were acquired over time $(t)$, the analyzed data had 5 dimensions $(x, y, z, t, \lambda)$. We detected fast movements of the ER that had escaped detection in previous studies with lower temporal resolution (Toresson and Grant, 2005). The prove-of-concept of automated analysis we present here allows us to scale up our experiments to large datasets containing thousands of spines, increasing the statistical power and reproducibility of morphometric studies.

\section{Methods}

In this section we introduce the concept and methods to successfully detect and segment spines. We discuss the conceptual idea of using statistical models of dendrite intensity and spine probability, the application to single time points and additional challenges in the analysis of time series. 


\subsection{Concept}

We use statistical dendrite intensity and spine probability models for spine prediction and detection. The analysis program was implemented in $\mathrm{C}++$, using $\mathrm{ITK}^{1}, \mathrm{VTK}^{2}$, and $\mathrm{QT}^{3}$. To generate a large set of training data, we reconstructed stretches of spiny dendrites from SBEM data and generated synthetic fluorescence images (SFI) by convolution with the point-spread-function (PSF). In addition, we computed SFI of spine structures only and resampled both datasets by backbone-orthogonal, registered slices (Fig. 1A). Next, we calculated spine probability maps from corresponding orthogonal SFI slices (Fig. 1B). Principal Component Analysis (PCA) models of dendrite intensity and of spine probability were computed (Blumer et al., 2011). These two statistical models incorporate the knowledge about intensity distributions that signal the presence of a dendritic spine. They have be generated only once for a specific microscope and cell type. If fluorescence data from a microscope with a very different PSF need to be analyzed, it is advisable to generate a new set of training data to calculate appropriate PCA models.

To detect spines in 3D fluorescence images, we also started by extracting 2D slices orthogonal to the backbone (Fig. 1C). These orthogonal slices were the basis of the spine prediction. They were approximated by the dendrite intensity model first; model coefficients were then transferred to the spine probability model to reconstruct 2D spine probability maps. Typically, individual spines contributed fluorescence to several adjacent slices. Therefore, we introduced additional backboneparallel features (Section 2.2.3). These features, which were computed over multiple slices (Fig. 2), ensured more robust detection results. The $2 \mathrm{D}$ prediction results were then projected back to $3 \mathrm{D}$ space and combined with the backbone-parallel features. Finally, a threshold was applied to binarize the computed 3D prediction map (Section 2.2.4). The resulting 3D objects correspond to spines or spine candidates for time series.

In the analysis of time series, we predicted spines independently at every time point. To enable analysis of individual spines over time, rigid registration of the input images was performed. Following spine prediction, the most probable spine paths were computed (2.2.5). The resulting spine paths correspond to the fluorescence intensity of individual spine heads over time, which, in the case of soluble (cytoplasmic) fluorescent proteins or dyes, is proportional to their volume.

\subsection{Segmentation using dendrite intensity and spine probability model}

Here we describe the detection and segmentation of spines using statistical models. We extend the previously described concept of computing SFIs, dendrite intensity and spine probability models (Blumer et al., 2011) to account for multiple spine orientations.

\subsubsection{Training of statistical models}

The statistical models of dendrite intensity and spine probability were based on SBEM data, the generation of which is described in section 3.1. We manually traced EM datasets to ensure that also spines with very thin necks were correctly reconstructed (Fig. S1). We computed SFIs by convolving the geometrically correct reconstruction with a Gaussian approximation of the PSF (Fig. 1A) (Zipfel et al., 2003; Zhang et al., 2010). SFIs were computed for dendrites including spines and for spines only. Dividing the SFI of spines by the SFI of the dendrite including spines resulted in 3D maps of spine

\footnotetext{
${ }^{1}$ ITK: Insight Segmentation and Registration Toolkit, www.itk.org

${ }^{2}$ VTK: Visualization Toolkit, www.vtk.org

${ }^{3}$ QT: User Interface Framework, www.qt-project.org
} 
probability. From the 3D probability map and the SFI of a dendrite, we extracted backboneorthogonal slices. To correct for the distortion in axial direction introduced by the elongated PSF, we registered every slice to a circular template (Fig. S2). We calculated 9 orientation-dependent dendrite intensity and spine probability PCA models (Fig. 1B). Eight models were computed having spines into one direction (radial $45^{\circ}$ segments) and the last model $(k=\{1, \ldots, 9\}, K:=9)$ represented a section without spine ( $<2.5 \%$ pixels with more than $50 \%$ spine probability). The number of radial segments was chosen to ensure that a spine occupies mainly one or two regions. Increasing the number of segments would reduce the power of each model to contain a complete description of the spine. With less segments, the risk that the model must be able to describe multiple spine orientations increases. The nine models were also used to improve the backbone position. Data $X_{d}$ (spiny dendrite intensity slices) and $X_{S}$ (spine probability maps) were divided into $X_{d}^{1}$ to $X_{d}^{9}$ and $X_{S}^{1}$ to $X_{s}^{9}$. In the matrices $X$, the examples are stored vectorized in the columns and the samples are mean-free (mean of all corresponding samples, representing fluorescence from the dendritic shaft for the intensity model, subtracted from each sample). For each orientation and model, the data was decomposed by using Singular Value Decomposition (SVD), resulting in $X=U D V^{T}$ where $U$ and $V$ are the unitary matrices with the Eigenvectors of $X X^{T}$ and $X^{T X}$ respectively. $D$ is a diagonal matrix with the corresponding Eigenvalues from $X X^{T}$ and $X^{T X}$ (which are the same) on its diagonal. We computed from the mean-free data the models $M_{d}^{1}$ to $M_{d}^{9}$ and $M_{s}^{1}$ to $M_{s}^{9}$. These models, which incorporate knowledge about the typical intensity distribution caused by spine in an image produced by a particular microscope, are the basis of our spine detection algorithm. Generation of a new set of models is only required in two cases: $(A)$ data from a microscope with a very different PSF has to be analyzed, or (B) study of a type of neuron with spines of very different shape or size.

\subsubsection{Backbone optimization}

In the current version of our program, we initialize the backbone by a list of manually clicked points $p_{i}=\left[\begin{array}{lll}x_{i} & y_{i} & z_{i}\end{array}\right]^{T}$ where $x_{i}$ and $y_{i}$ are specified by the user in a volume rendering viewed from top. The depth $z_{i}$ is automatically set to the point of maximum intensity at position $x_{i}, y_{i}$. The points $p_{i}$ are located in sequence along the dendrite such that a B-spline gives a first approximation of the backbone. We can specify multiple line parts to approximate multiple backbone pieces, represented by separate B-splines. Thus, several regions of interest (stretches of dendrite) can be analyzed at once. Every B-spline is uniformly sampled at regular intervals and backbone-orthogonal 2D slices are extracted at the given positions. The intensity in the slices is normalized and the slices themselves are registered to the template used for the model computation. Dendritic spines can introduce errors (lateral shift) in the backbone generation procedure. To address this problem, we extracted additional slices $s_{d, i, j}$ shifted parallel to the slices $s_{d, i}$ (in the slice plane) at the sampled B-spline positions. Thus, for each sampled B-spline position, there exists a number of intensity-normalized and registered slices $s_{d, i, j}$. For each slice, the probability of good representation of the slice by a particular dendrite intensity model can be computed by:

$$
P\left(s_{d, i, j}, M_{d}^{k}\right)=P\left(s_{d, i, j} \mid M_{d}^{k}\right) P\left(M_{d}^{k}\right)
$$

\section{Equation 1}

To find the most probable slices $s_{d, i, j}$, of the shifted slices $s_{d, i, j}$ over all sampled B-spline positions, we selected the maxima:

$$
j^{\prime}=\underset{j}{\operatorname{argmax}}\left\{P\left(s_{d, i, j}, M_{d}^{k}\right)\right\}
$$


Over the most probable slices $s_{d, i, j}$, and their 3D locations, a new B-spline was approximated. We extracted new seed positions by taking the location with the highest probability and removed all neighboring positions within a given radius. Thus, the new B-spline was defined by the most probable locations, avoiding registration errors introduced by statistically unlikely slice positions.

\subsubsection{Spine prediction}

To predict the presence of spines in synthetic or real fluorescence images (Fig. 1C), we started by extracting test slices $s_{d, i}$ orthogonal along the dendritic backbone, identical to the training procedure (Section 2.2.1). Test slices were resisted to a circular template to correct for distortion (Fig. S2).

A slice $s_{d, i}$ was approximated by the model $M_{d}^{k}$ by

$$
s_{d, i} \approx M_{d}^{k}\left(\alpha_{d, i}^{k}\right)=\mu_{d}^{k}+U_{d}^{k} \alpha_{d, i}^{k}
$$

Equation 3

After extraction of the coefficients $\alpha_{d, i}^{k}$ by approximation with the dendrite intensity model, coefficients were transferred to the spine probability model to compute the coefficients $\alpha_{s, i}^{k}$. As introduced in (Blumer et al., 2011) the coefficients are inferred by:

$$
\alpha_{s, i}^{k}=D_{s}^{k} V_{s}^{k^{T}} V_{d}^{k} D_{d}^{k} \alpha_{d, i}^{k}
$$

\section{Equation 4}

For each test slice, we reconstructed 9 spine probability maps $s_{s, i}^{1}$ to $s_{s, i}^{9}$ using all models. The models $M_{d}^{9}$ and $M_{s}^{9}$ had no spine contribution and the prediction maps of these models were ignored.

A limitation of 2D spine probability maps is the lack of information in the third dimension, along the dendritic backbone. To overcome the independence of the backbone-orthogonal slices and increase the reliability of spine prediction, we computed backbone-parallel features in the slices $s_{d, i}$ (Fig $2 \mathrm{~A}$ ). The feature value indicates intensity changes measured parallel to the backbone. If a spine is present, intensity along the dendrite show a characteristic peak (Fig. 2B and C). For each pixel in every 2D slice, we computed a feature value in the registered and unregistered $2 \mathrm{D}$ slices $s_{d, i}$ and $\tilde{s}_{d, i}$ respectively (Fig. 2D). As spines vary greatly in intensity, we used local gradients rather than absolute values. Depending on the size and orientation of the spine, it is likely to be sampled in multiple slices. Therefore, we require a variable distance to calculate the feature. Within a local search region, we used the minimum and normalized it by the intensity of the pixel for which we computed the feature. For a pixel $(x, y)$ of slice $s_{d, i}$ the feature is computed by:

$$
\operatorname{bpf}\left(s_{d, i}(x, y)\right)=\frac{s_{d, i}(x, y)-\min _{l=(1, \ldots, L)} s_{d, i-l}(x, y)}{s_{d, i}(x, y)}+\frac{s_{d, i}(x, y)-\min _{l=(1, \ldots, L)} s_{d, i+l}(x, y)}{s_{d, i}(x, y)}
$$

Equation 5

Backbone-parallel features $b p f(\ldots)$ were computed in the space of registered slices $s_{d, i}$ and corresponding unregistered slices $\tilde{s}_{d, i}$, designated $b p f_{r}$ and $b p f_{u}$, respectively. 
Next, we combined information from the BPFs from registered and unregistered slices and the spine probability maps in all (eight) directions to produce cumulative spine prediction maps. For each pixel the three values were composed together with additional weights to prediction maps $\mathrm{pm}$ :

$$
p m_{i, k}(x, y)=b p f_{u}\left(\tilde{s}_{d, i}(x, y)\right) b p f_{r}\left(s_{d, i}(x, y)\right) s_{s, i}^{k}(x, y) \omega_{\gamma} \omega_{s} \omega_{s K}
$$

Equation 6

The weights represent how strongly the prediction depends on the orientation of the slice $\left(\omega_{\gamma}\right)$ and the probability that the slice contains a spine at all $\left(\omega_{s}\right.$ and $\left.\omega_{s K}\right)$. The weight of orientation of the slice $\left(\omega_{\gamma}\right)$ corresponds to the reliability of the prediction relative to the training data. The models are currently not very reliable for horizontally oriented slices (i.e. vertical dendrites), as they were trained mainly with horizontally oriented dendritic sections which are dominant in organotypic slice cultures. The weight is computed by

$$
\omega_{\gamma}\left(\mathrm{s}_{\mathrm{d}, \mathrm{i}}\right)=1-\left|\left\langle\vec{n}_{i}, \vec{z}\right\rangle\right|=1-\left|n_{i, z}\right|
$$

Equation 7

where $\vec{n}_{i}$ is the normal of the extracted slice and $\vec{z}$ the optical axis of the objective. The weight $\omega_{S}$ is the probability of having a slice containing a spine. It is computed by:

$$
\omega_{s}\left(\mathrm{~s}_{\mathrm{d}, \mathrm{i}}\right)=\mathrm{P}\left(\mathrm{s}_{\mathrm{d}, \mathrm{i}}=\text { spine }\right)=1-\frac{P\left(s_{d, i}, M_{d}^{K}\right)}{\sum_{k=1}^{K} P\left(s_{d, i}, M_{d}^{k}\right)}
$$

\section{Equation 8}

The last weighting vector $\omega_{s K}$ depends on the orientation-dependent model. Therefore, eight versions exist which depend on the selected model $(k)$. Instead of the original weight, the weight $\omega_{S K}$ is a normalized version. We normalized the weight to the range $[0,1]$ between local minima and maxima. The non-normalized weight $\omega_{S K}^{\prime}$ is computed by:

$$
\omega_{s K}^{\prime}\left(s_{d, i}, k\right)=\frac{P\left(s_{d, i}, M_{d}^{k}\right)}{P\left(s_{d, i}, M_{d}^{k}\right)+P\left(s_{d, i}, M_{d}^{K}\right)}
$$

Equation 9

The orientation-dependent prediction maps $p m_{i, k}$ are combined to a final orientation-independent prediction map $\mathrm{pm}_{i}$ by applying a pixel-wise maximum operator:

$$
p m_{i}(x, y)=\max _{k}\left\{p m_{i, k}(x, y)\right\}
$$

\section{Equation 10}

The application of the pixel-wise maximum operator combines positive spine prediction results over all model directions. The combination of multiple features (model prediction and backbone-parallel features) and the weights proved to be robust in practical use. Robust detection and segmentation are essential for a low rate of false positives, i.e. spurious spines. The introduction of backboneparallel features increased precision, especially in locations where correct registration of the slices was difficult due to strong curvature of the backbone. 


\subsubsection{Binarization}

At the end of spine detection, we needed a clear yes/no decision. Therefore, the spine prediction map had to be binarized, which can be done in a global fashion or locally adaptive. We used a locally adaptive approach as we had spines with weak and strong detection results. The locally adaptive approach detected all local maxima and computed for each maximum a local thresholding value, relative to the amplitude of the local maximum. The local threshold was applied inside a search window around the local maximum. More complex thresholding algorithms (e.g. Otsu thresholding) can be used, but did not improve the results significantly. In our application, we used a relative threshold $t=c \max _{L}(\mathrm{pm})$ where $L$ is the local window and $\mathrm{pm}$ the 3D prediction map. In practice, a suitable value was $c=0.7$. If seed points are closely spaced, the search windows can overlap. To separate spines, all voxels belonging to multiple search windows had to be assigned to a specific spine. In most cases, a distance criterion could be used to make this decision: Each voxel was assigned to the closest local maximum.

\subsubsection{Time series analysis}

Automated analysis of time series poses additional challenges for spine detection and segmentation. To compensate potential translation and rotation induced by drift of the tissue between time points, $3 \mathrm{D}$ registration is required prior to analysis. Manual backbone initialization for multiple time points is too time-consuming. Therefore, we implemented automatic initialization of the backbone based on the first time point of a series. Finally, each spine must be identified in each time point to enable automated tracking of spine changes over time. Tracking of individual spines is required because spines in live tissue show constant micro-movements and dynamic changes in neck length.

We used a rigid registration algorithm to compensate for translation $(x, y$ and $z)$ and rotation (around $x-, y-$, and $z$-axis). Each time point was registered to the first point of the series. In practice, rotation was minimal and limited to the $z$-axis, as the sample was placed on the level glass bottom of a recording chamber. We find for each input images $I_{t}$ with $t>1$ the following optimal transformation $M_{t}^{*}$ by:

$$
\mathrm{M}_{\mathrm{t}}^{*}=\underset{M}{\operatorname{argmin}} \sum_{\Omega}\left(M\left(I_{t}\right)-I_{1}\right)^{2}
$$

\section{Equation 11}

where $M$ is the transformation consisting of a translation and rotation and $\Omega$ is the image domain. The registered input images made it possible to initialize the backbone only for the first time point. Furthermore, instead of a complex tracking of spines, a simpler search of spine paths by path cost was established. From the spines of the first time point over all other time points and their spines, all possible paths were computed. To each path, a cost-factor was assigned, composed of the distance and detection probability. Finally, repeatedly the cheapest path was selected as spine path and conflicting ones removed from the set of possible paths. Every spine path corresponds to the trace of a single spine over time. The cost of the spine path was defined as the distance and likelihood of each spine to its preceding spine and to the first spine of the path:

$$
C\left(S_{1, q_{1}}, \ldots, S_{T, q_{T}}\right)=\sum_{t=1}^{T}\left\{\frac{\left\|p\left(S_{t, q_{t}}\right)-p\left(S_{t-1, q_{t-1}}\right)\right\|}{L\left(S_{t, q_{t}}\right) L\left(S_{t-1, q_{t-1}}\right)}+\frac{\left\|p\left(S_{t, q_{t}}\right)-p\left(S_{1, q_{1}}\right)\right\|}{L\left(S_{t, q_{\mathrm{t}}}\right) L\left(S_{1, q_{1}}\right)}\right\}
$$


where $S_{t, q_{t}}$ is the $q_{t}$ th spine candidate at time point $t, L(\ldots)$ is the likelihood from the 3D prediction map and $p(\ldots)$ is the position of the spine in $3 \mathrm{D}$. The first summand minimizes the movement from time point to time point. The second summand restricts the movement away from the first time point. As all images were registered, there was little movement over time. Taking into account differences in likelihood prefers well-detected spines over weak ones.

A precisely positioned dendrite backbone proved to be essential for the detection and prediction of spines using statistical models. To optimize the backbone starting from the manual initialization, we also used the statistical models (Section 2.2.2). Combined with the registration of time series data to the first time point, we were able to overcome the issue of backbone initialization for each time point.

\section{Results and discussion}

In this section, we report the performance of our spine detection algorithm using correlative datasets and show biological applications in multi-channel images and time series. Initially, we used SBEM reconstructions of two sections of spiny dendrites from two different neurons to train the statistical models. The resulting model was tested on two independent correlative light/electron microscopy (CLEM) datasets from two different CA1 pyramidal cell branches. To analyze our application examples (Fig. 5 and 6), we generated an improved statistical model based on all four SBEM datasets (Fig. S1). Automatic detection and segmentation of spines in single images and time series was possible on standard hardware. Typical images sizes ( $512 \times 512 \times 30$ voxels) were analyzed on current personal computers (e.g. Intel (R) Core (TM) i7-870 at $2.93 \mathrm{GHz}$ and 16 GB RAM) in about $5 \mathrm{~min}$. Practical tests demonstrated that time series with 20 time points and image size of $1024 \times 1024 \times 170$ voxels could be processed on the same hardware.

\subsection{Correlative light/electron microscopy dataset}

Previous attempts to detect dendritic spines in fluorescence images have used manual and automatic analysis of the same dataset as a benchmark (e.g. (Yuan et al., 2009; Rodriguez et al., 2007; Zhang et al., 2007)). Due to the diffraction-limited and non-isotropic performance of light microscopes, this approach is quite problematic: Very thin or stubby spines, or spines leaving the dendrite in $z$ direction along the optical axis, might generate a signal that is not detectable by even the most experienced human observer. Although this form of benchmarking leads to impressive performance figures for the automated detection programs, here we are interested in another number: What fraction of the complete set of existing spines can be detected in fluorescence images?

To address this question, we generated correlative light and electron microscopy (CLEM) datasets, imaging the same spiny dendrite first live with a two-photon microscope and subsequently with a serial block-face scanning electron microscope (SBEM). A similar approach using serial section transmission electron microscopy has been used in many in vivo studies to confirm by EM the presence of synapses on spines observed in the living mouse brain (Trachtenberg et al., 2002; Holtmaat et al., 2005; Knott et al. 2009). The two-photon microscope was based on an Olympus BX61-WI microscope with a 60x 0.9 NA water immersion objective and two-color detection (Oertner, 2002), controlled by ScanImage (Pologruto et al., 2003). The scanning electron microscope (Quanta $200 \mathrm{FEG}, \mathrm{FEI}$ ) was equipped with an ultra-microtome (3View, Gatan) in order to cut and image automatically inside the microscope. The segmentation problem posed by the very large SBEM datasets was solved by photoconversion of neurobiotin, which made the chosen neuron highly 
electron-dense and generated strong contrast to the surrounding tissue. The strong contrast made it possible to perform a first pass 3D reconstruction using a simple thresholding operation (Imaris, Bitplane AG). The result of this automatic segmentation procedure had imperfections, however, as thin-necked spines were frequently detached from the dendrite and the dendritic surface contained holes. Therefore, we resorted to manual tracing of SBEM data to generate the correlative datasets used in this study (Fig. S1), putting us in the unique situation to compare the performance of automated spine detection on fluorescence images to ground truth data from the very same dendritic section.

To generate additional training data for the statistical spine model, we used a number of SBEM reconstructions from neurons that were not previously imaged live (Fig. S1). These 3D reconstructions were convolved with the PSF to generate synthetic fluorescence images (SFI). The principle of generating training data and train the models is introduced in section 2.2 and (Blumer et al., 2011). Automatic spine detection was then tested on independent correlative datasets, starting with SFIs (Fig. 3A). On SFIs, detection precision was very high (Fig. 3A bottom, no false positives), reflecting the fact that no photon- or background noise was added when generating these images. Spines protruding downwards, however, were frequently missed (poor recall) due to strong low-pass filtering along the $z$-axis of the PSF (Fig. 4A). This illustrates a physical limitation of traditional diffraction-limited microscopy that can only be cured by reducing the size of the PSF, e.g. by STED microscopy (Nägerl et al., 2008; Takasaki et al., 2008; Testa et al., 2012). In the real 2PM images of the same dendritic section, recall was comparable, but several false-positive spines were detected in the background (low precision, Fig. 3B middle). Using slightly higher excitation laser power, false positives could be completely avoided (Figs. 5 and 6). Thus, precision depends on the signal-to-noise ratio of the $2 p$ images as well as the threshold used for binarization of the spine probability map and is not an inherent problem of our spine detection strategy. Similar results were obtained on a second CLEM dataset (Table 1, Fig. S1). For 2PM data, we achieved a precision and recall of about 0.8 , which might seem low compared to previous publications. However, our result takes into account all spines that exist, including spines pointing toward the $z$-direction. Therefore, the precision and recall figures in this study are not directly comparable to the benchmark procedure of other studies.

\begin{tabular}{llllllll}
\hline Data Source & Dataset & \# Spines & TP & FP & FN & Precision & Recall \\
\hline $\begin{array}{l}\text { Synthetic } \\
\text { fluorescence } \\
\text { images based on }\end{array}$ & Dendrite \#4 & 15 & 10 & 3 & 5 & 0.77 & 0.67 \\
SB-EM & Dendrite \#6 & 16 & 11 & 0 & 5 & 1.00 & 0.69 \\
& Cumulative & $\mathbf{3 1}$ & $\mathbf{2 1}$ & $\mathbf{3}$ & $\mathbf{1 0}$ & $\mathbf{0 . 8 8}$ & $\mathbf{0 . 6 8}$ \\
\hline $\begin{array}{l}\text { Two-photon } \\
\text { microscopy of live } \\
\text { neuron }\end{array}$ & Dendrite \#4 & 15 & 13 & 3 & 2 & 0.81 & 0.87 \\
& Dendrite \#6 & 16 & 12 & 4 & 4 & 0.75 & 0.75 \\
& Cumulative & $\mathbf{3 1}$ & $\mathbf{2 5}$ & $\mathbf{7}$ & $\mathbf{6}$ & $\mathbf{0 . 7 8}$ & $\mathbf{0 . 8 1}$ \\
\hline
\end{tabular}

Table 1: Quantitative results of automatic spine detection on two CLEM datasets. TP, true positives; FP, false positives; FN, false negatives. Precision is the fraction of detected spines that are relevant. Recall is the probability that an existing spine will be detected.

The false positive rate in the analysis of 2PM data was higher than for SFI data, chiefly for two reasons: First, to generate SFI images, we used a simple Gaussian approximation of the PSF. In 
reality, the PSF is more complex and includes side maxima that further degrade the image. Second, dim structures like dendritic spines suffer from photon shot noise and dark noise of the photomultiplier tubes. These noise sources were not simulated when we generated SFI images. Still, we identified one problem that produced false positives even in noise-free SFI data: Correct placement of the dendritic backbone was critical for the spine detection process. If the backbone moves toward one side of the dendritic shaft, for example due to excessive smoothing of a sharp bend in the dendrite, false positive detections could occur at the outside corner of the bend. Thus, the second round of backbone optimization based on the orthogonal slices was important for a reliable result. Detection precision is less of a concern in time series analysis, because spurious detections in single time points are efficiently filtered out in the process of spine registration over time. As automatic analysis of time series is of particular interest to us, we optimized system parameters to perform well for time series and used the same settings for the analysis of the correlative data. We were surprised about the number of false negatives in the analysis of SFI data, including some small spines that were correctly detected in the real two-photon images. The threshold for binarization of spine probability maps was identical in all analyses. It is possible that fluorescent dye molecules had accumulated in spines, leading to a stronger fluorescence signature than expected from the convolution of spine head volume with the PSF. Further correlative studies will be needed to investigate the distribution of different dyes in neurons.

Having confirmed our suspicion that small spines protruding in z-direction are impossible to detect in real or synthetic fluorescence images, we wanted to know the likelihood of such a spine orientation. Based on our EM data, we could show that spines in organotypic slice cultures rarely point upwards (Fig. 4B and C). Thus, the fraction of 'invisible spines' in $2 p$ data (false negatives, $19 \%$ ) is actually lower than would be expected for random spine orientation, and most missed spines are indeed hidden below the dendrite (Fig. 4A).

\begin{tabular}{llllllll}
\hline Software & Dataset & \#Spines & TP & FP & FN & Precision & Recall \\
\hline NeuronIQ 2.0 & Dendrite \#4 & 15 & 10 & 3 & 5 & 0.77 & 0.67 \\
& Dendrite \#6 & 16 & 7 & 6 & 9 & 0.54 & 0.44 \\
& Cumulative & $\mathbf{3 1}$ & $\mathbf{1 7}$ & $\mathbf{9}$ & $\mathbf{1 4}$ & $\mathbf{0 . 6 5}$ & $\mathbf{0 . 5 5}$ \\
\hline
\end{tabular}

Table 2: Quantitative results of automatic spine detection on 2PM data using NeuronIQ 2.0. The results can be compared to the 2PM data of table 1.

For comparison, we analyzed our 2PM data with NeuronIQ TE (He et al., 2012). As this software requires higher oversampling in z-direction, we resampled our original images accordingly. We tested the parameter domain extensively, but found that default settings generated the best results. Precision was similar to our approach for Dendrite \#4, but worse for Dendrite \#6. Recall was worse for both datasets (Table 2, Fig. S3A). The commercial software IMARIS (Bitplane AG) contains a module for automated tracing of filaments, but had problems distinguishing spines from dendritic side branches (Fig. S3B). On images with higher contrast, the performance difference might have been less dramatic. In live cell imaging, however, high contrast comes at the cost of increased fluorophore bleaching and photodamage, which are major limiting factors in time-lapse studies. The 
better performance of our approach on noisy datasets highlights the power of using statistical models.

Using CLEM data to benchmark spine detection software is a novel approach, and our results suggest that for any software, precision and recall strongly depend on spine orientation. We believe that in contrast to expert labeled fluorescence data, detection of z-oriented spines can be studied much better if ground truth is available. Thus, to promote further developments in the field, our CLEM datasets are available for academic use at http://www.spinedetection.com.

\subsection{Automatic detection of synaptically connected spines}

To apply and further test our spine detection software, we used a dataset where spiny dendrites and active presynaptic terminals from different neurons were labeled with fluorescent proteins of different color (Fig. 5A). A particular challenge was the automatic detection of those spines that were in direct contact with a presynaptic terminal. Due to the diffraction-limited spatial resolution of the two-photon microscope, structures within the radius of the point-spread-function $(0.6 \times 0.6 \times 1.6 \mu \mathrm{m})$ cannot be separated optically. In this application, we used the blurring caused by the PSF to our advantage: We reasoned that red fluorescence from an adjacent presynaptic terminal should be detectable in the voxels assigned to a (green fluorescent) dendritic spine. First, we automatically detected and segmented all spines on a stretch of dendrite using the volume channel (Fig. 5B). For each spine, we plotted postsynaptic green fluorescence (spine volume) versus presynaptic red fluorescence (Fig. 5C). In the example presented here, spine \#5 is the only spine that exceeds an arbitrary threshold of 2 fluorescence units in the red channel. Therefore, it is potentially in functional contact with a labeled presynaptic terminal.

As we had co-expressed a light-gated channel in the presynaptic axon and calcium-sensing fluorescent protein (GCaMP3) in the postsynaptic cell, we could compare the result of our automated proximity detection to the results of functional imaging. Indeed, short pulses of blue light induced calcium signals in spine \#5, but not in any other spine on this stretch of dendrite (Fig. 5D), indicating that this and only this spine received glutamate from a light-activated axon. For a more detailed description of these optogenetic experiments, please see (Wiegert and Oertner, 2013). Simultaneous analysis of multiple color channels opens a wide range of applications, including ratiometric measurements of protein concentration in individual spines (Zhang et al., 2008). Here we show that automated analysis of pre- and postsynaptic labels is possible, allowing us to successfully identify spines that receive synaptic input from a defined subset of axons.

\subsection{Automated analysis of organelle motility in time series}

Live cell imaging is a powerful technique as it allows following biological processes over time. Good temporal resolution is particularly important to understand the highly dynamic processes that shape the brain. Quantitative analysis of time series, however, is notoriously difficult, as living biological tissue is never quite as stable as fixed preparations. Manual analysis of such datasets is extremely time consuming and often the limiting factor in terms of throughput and temporal resolution. Here we present an example of automatic analysis of individual spines in 5D (3 spatial dimensions, 2 colors, and time) to track the dynamics of an intracellular organelle, the endoplasmic reticulum (ER). 
The ER forms a dynamic network inside every neuron (Toresson and Grant, 2005). Spines containing ER tend to have strong synapses and are preferential sites for a particular type of synaptic plasticity, mGluR-dependent long-term depression (Holbro et al., 2009). How long ER typically resides in individual spines is poorly understood. The goal of this project was the automatic detection of ER as it invades individual dendritic spines and an analysis of its temporal dynamics. Individual neurons were co-transfected with ER-targeted GFP and cytoplasmic dsRed to visualize the entire volume of the cell (Holbro et al., 2009). Image stacks were taken every $10 \mathrm{~min}$ at a resolution of $0.063 \mu \mathrm{m} / \mathrm{pixel}$ in $x y$ and $0.3 \mu \mathrm{m} /$ pixel in $z$-direction. Lateral drift ( $2-5 \mu \mathrm{m} / \mathrm{h}$, typically) was compensated by refocussing and by post-hoc 3D alignment (2.2.5). We started by automatic detection and segmentation of spine candidates in each time point, using the red volume signal (Fig. 6A). In the tree of all spine candidates, the most probable path over all time points was determined. Each path corresponds to a spine and the path nodes to the different time points of the corresponding spine. In the example, 10 spines were successfully detected, segmented and tracked over all time points (precision 1.0; recall 1.0). For each spine, all voxels were sorted by intensity and the brightest $5 \%$ were averaged in both color channels (Fig. 6B, two examples). Spine \#5 showed significant increase of green fluorescence at time points 4,8 and 16, indicating transient invasions of ER. A correlation between ER invasion and spine volume was not obvious in this example. Spine \#6 was apparently never invaded by ER, as no green fluorescence maxima were detected over time. Inspection of the input images (Fig. 6C) corroborated the result of the automatic analysis. This application example demonstrates that it is feasible to detect, segment and track the volume of spines over time and to monitor the presence or absence of intracellular organelles in an automated fashion. Even this single experiment contains 180 regions of interest that have different shapes and sizes; manual analysis would have been tedious. At a lower temporal sampling rate, more suitable for manual analysis, the brief visitations of ER into individual spines could have easily escaped detection. Applying automatic detection to longer stretches of dendrite allowed us to analyze dwell times of ER in spines, which were highly variable (Fig. 6D). Most visits lasted less than $10 \mathrm{~min}$, but some spines were permanently occupied by ER (Fig. 6E). Within 5 h, $88 \%$ of spines were visited by ER at least once, a surprisingly high number. As calcium release from the ER is thought to be important for several forms of synaptic plasticity (Jedlicka et al., 2008), it will be interesting to compare the volume of spines before, during, and after ER invasion. Picking up subtle changes in spine volume in a highly variable population requires analysis of a large number of individual spines across multiple time points. As this example shows, reliable spine detection and tracking over time are necessary ingredients to extract biologically relevant information from images of neuronal structure.

\section{Conclusion}

We present a machine leaning approach to automatically segment dendritic spines in two-photon microscopy data and to trace their fate over time. Our spine detection algorithm is based on the computation of statistical dendrite intensity and spine probability models. To generate a large amount of training data for the algorithm, we introduced a new method for the generation of synthetic fluorescence images (SFI) based on automated SBEM reconstructions of dendritic geometry. In the training process, we require no manual classification of spines in the fluorescence domain, which is error-prone due to poor z-resolution. Instead, spines were annotated in surface models of EM reconstructions, a process that requires no special expertise due to the excellent spatial resolution of SBEM. To test the performance of our spine detection software, we generated correlative two-photon/EM datasets. Automatic spine analysis of two-photon data was compared to 
ultrastructural information about the presence or absence of spines. As expected, spines oriented along the optical axis of the microscope were not detectable in fluorescence datasets. On the bright side, we report that these spines are underrepresented in organotypic slice cultures. Our results suggest that about $20 \%$ of spines will be missed in the analysis (manual or automatic) of horizontally oriented dendrites in diffraction-limited 2PM datasets.

Our introduction of statistical models for spine detection had two major consequences: First, no human expert was needed to label putative spines in fluorescence datasets. Second, spines of typical size and shape (i.e. spines that were frequent in the training dataset) were detected best. Thus, the program had an inherent bias against detection of non-spines (e.g. filopodia, dendritic side branches, and fluorescent debris). In the current version of our software, manual initialization of the backbone allows the user to control which dendritic section should be analyzed. This was a useful feature for our application, but it prevented fully automatic analysis and batch processing. Sophisticated algorithms for automatic backbone reconstruction of neurons in fluorescence images have been developed (González et al., 2009; Wang et al., 2011; Chothani et al., 2011), and the Diadem challenge provides a useful benchmarking procedure for this computational problem. Similar to the processing of time series, it would be feasible to initialize our spine detection software with the result of a dendrite tracing algorithm. In future extensions, integration of a dendrite tracing algorithm to initialize spine detection could be a powerful strategy to analyze all spines on a neuron.

In the second part of our study, we applied our spine detection program to address two biological problems: First, we performed automatic detection of spines that are in functional contact with labeled presynaptic terminals. Second, we tracked the presence or absence of intracellular organelles in individual dendritic spines over multiple time points. As our software proved to be useful in these applications, it allows us to scale up our experiments from the proof-of-concept level shown here to a scale that is limited by the speed of data acquisition, not data analysis.

With the advent of high-throughput imaging techniques such as spinning disc microscopy, SPIM etc., analysis of vast amounts of data has become a severe bottleneck, limiting the statistical power of many studies today. We are convinced that automatic segmentation and analysis of dendritic spines will open new possibilities for neurobiological studies, simplifying e.g. the analysis of animal models of human mental disorders. Manual analysis is not only tedious, but also hard to compare between laboratories. The greater statistical power that comes with automated image analysis will make it possible to detect subtle changes in brain microanatomy that might have escaped previous studies.

\section{Acknowledgements}

We would like to thank Daniela Gerosa-Erni for excellent technical assistance. This work was supported by the Novartis Research Foundation, Neurex, EMBO, and SystemsX.ch. 


\section{References}

Blanz, V., Vetter, T., 1999. A Morphable Model for the Synthesis of 3D Faces. Siggraph 99, 187-194.

Blumer, C., Vivien, C., Oertner, T. G., Vetter, T., 2011. Fluorescence Image Segmentation by Using Digitally Reconstructed Fluorescence Images. IN Proc. of Microscopic Image Analysis with Applications in Biology - MIAAB, Heidelberg, 1-5.

Briggman, K. L., Denk, W., 2006. Towards neural circuit reconstruction with volume electron microscopy techniques. Current Opinion in Neurobiology 16 (5), 562-570.

Brown, K. M., Barrionuevo, G., Canty, A. J., De Paola, V., Hirsch, J. A., Jefferis, G. S. X. E., Lu, J., Snippe, M., Sugihara, I., Ascoli, G. A., 2011. The DIADEM data sets: representative light microscopy images of neuronal morphology to advance automation of digital reconstructions. Neuroinformatics, 9 (2-3), 143-157.

Cheng, J., Zhou, X., Miller, E., Witt, R. M., Zhu, J., Sabatini, B. L., Wong, S. T. C., 2007. A novel computational approach for automatic dendrite spines detection in two-photon laser scan microscopy. Journal of Neuroscience Methods 165 (1), 122-134.

Chothani, P. , Mehta, V., Stepanyants, A., 2011. Automated Tracing of Neurites from Light Microscopy Stacks of Images. Neuroinformatics 9 (2-3), 263-278.

Cootes, T. F., Taylor, C. J., Cooper, D. H., Graham, J., 1995. Active Shape Models - Their Training and Application. Computer Vision and Image Understanding 61 (1), 38-59.

Denk, W., Horstmann, H., 2004. Serial block-face scanning electron microscopy to reconstruct threedimensional tissue nanostructure. PLoS Biology 2 (11), e329.

Fehr, J., 2009. Local invariant features for 3D image analysis. PhD Thesis, Albert-Ludwigs-Universität Freiburg, Germany.

Gala, R., Chapeton, J., Jitesh, J., Bhavsar, C., Stepanyants, A. , 2014. Active learning of neuron morphology for accurate automated tracing of neurites. Frontiers in Neuroanatomy 8 (37), 1-14.

Gilette, T. A., Brown, K. M., Ascoli, G. A., 2011. The DIADEM metric: comparing multiple reconstructions of the same neuron. Neuroinformatics 9 (2-3), 233-245.

González, G., Aguet, F., Fleuret, F., Unser, M., Fua, P., 2009. Steerable Features for Statistical 3D Dendrite Detection. IN Medical Image Computing and Computer-Assisted Intervention - MICCAI, London, 625-632.

He, T., Xue, Z., Wong, S. T. C., 2012. A novel approach for three dimensional dendrite spine segmentation and classification. SPIE Medical Imaging 8314, 831437-1-831437-8.

Holbro, N., Grunditz, A., Oertner, T. G., 2009. Differential distribution of endoplasmic reticulum controls metabotropic signaling and plasticity at hippocampal synapses. Proceedings of the National Academy of Sciences USA 106 (35), 15055-15060.

Holtmaat, A. J. G. D., Trachtenberg, J. T., Wilbrecht, L., Shepherd, G. M., Zhang, X., Knott, G. W., Svoboda, K., 2005. Transient and persistent dendritic spines in the neocortex in vivo. Neuron 45 (2), 279-291. 
Janoos, F., Mosaliganti, K., Xu, X., Machiraju, R., Huang, K., Wong, S. T. C., 2009. Robust 3D reconstruction and identification of dendritic spines from optical microscopy imaging. Medical Image Analysis 13 (1), 167-179.

Jedlicka, P., Vlachos, A., Schwarzacher, S. W., Deller, T., 2008. A role for the spine apparatus in LTP and spatial learning. Behavioural Brain Research 192 (1), 12-19.

Knott, G. W., Holtmaat, A., Trachtenberg, J. T., Svoboda, K., Welker, E., 2009. A protocol for preparing GFP-labeled neurons previously imaged in vivo and in slice preparations for light and electron microscopic analysis. Nature Protocols 4 (8), 1145-1156.

Koh, I. Y. Y., Lindquist, W. B., Zito, K., Nimchinsky, E. A., Svoboda, K., 2002. An Image Analysis Algorithm for Dendritic Spines. Neural Computation 14 (6), 1283-1310.

Matsuzaki, M., Ellis-Davies, G. C. R., Nemoto, T., Miyashita, Y., lino, M., Kasai, H., 2001. Dendritic spine geometry is critical for AMPA receptor expression in hippocampal CA1 pyramidal neurons. Nature Neuroscience 4 (11), 1086-1092.

Nägerl, U. V., Willig, K. I., Hein, B., Hell, S. W., Bonhoeffer, T., 2008. Live-cell imaging of dendritic spines by STED microscopy. Proceedings of the National Academy of Sciences USA 105 (48), 1898218987.

Nimchinsky, E. A., Sabatini, B. L., Svoboda, K., 2002. Structure and function of dendritic spines. Annual Review of Physiology 64 (1), 313-353.

Oertner, T. G., 2002. Functional imaging of single synapses in brain slices. Experimental Physiology 87 (6), 733-736.

Pologruto, T. A., Sabatini, B. L., Svoboda, K., 2003. Scanlmage: flexible software for operating laser scanning microscopes. Biomed Eng Online 2 (1), 13.

Rodriguez, A., Ehlenberger, D. B., Dickstein, D. L., Hof, P. R., Wearne, S. L., 2007. Automated ThreeDimensional Detection and Shape Classification of Dendritic Spines from Fluorescence Microscopy Images. PLoS One 3 (4), e1997.

Takasaki, K. T., Ding, J. B., Sabatini, B. L., 2013. Live-Cell Superresolution Imaging by Pulsed STED TwoPhoton Excitation Microscopy. Biophysical Journal 104 (4), 770-777.

Testa, I., Urban, N. T., Jakobs, S., Eggeling, C., Willig, K. I., Hell, S. W., 2012. Nanoscopy of living brain slices with low light levels. Neuron 75 (6), 992-1000.

Toresson, H., Grant, S. G. N., 2005. Dynamic distribution of endoplasmic reticulum in hippocampal neuron dendritic spines. European Journal of Neuroscience 22 (7), 1793-1798.

Trachtenberg, J. T., Chen, B. E., Knott, G. W., Feng, G., Sanes, J. R., Welker, E., Svoboda, K., 2002. Long-term in vivo imaging of experience-dependent synaptic plasticity in adult cortex. Nature 420 (6917), 788-794.

Wang, Y., Narayanaswamy, A., Tsai, C.-L., 2011. A Broadly Applicable 3-D Neuron Tracing Method Based on Open-Curve Snake. Neuroinformatics 9 (2-3), 193-217.

Wiegert, J. S., Oertner, T. G., 2013. Long-term depression triggers the selective elimination of weakly integrated synapses. Proceedings of the National Academy of Sciences USA 110 (47), E4510-E4519. 
Xu, X., Cheng, J., Witt, R. M., Sabatini, B. L., Wong, S. T. C., 2006. A shape analysis method to detect dendritic spine in 3D optical microscopy image. Proceedings of International Symposium on Biomedical Imaging, 554-557.

Yuan, X., Trachtenberg, J. T., Potter, S. M., Roysam, B., 2009. MDL Constrained 3-D Grayscale Skeletonization Algorithm for Automated Extraction of Dendrites and Spines from Fluorescence Confocal Images. Neuroinformatics 7 (4), 213-232.

Zhang, Y., Zhou, X., Witt, R. M., Sabatini, B. L., Wong, S. T. C., 2007. Automated Spine Detection Using Curvilinear Structure Detector and LDA Classifier. Proceedings of International Symposium on Biomedical Imaging, 528-531.

Zhang, Y.-P., Holbro, N., Oertner, T. G., 2008. Optical induction of plasticity at single synapses reveals input-specific accumulation of $\alpha$ CaMKII. Proceedings of the National Academy of Sciences USA 105 (33), 12039-12044.

Zhang, B., Zerubia, J., Olivo-Marin, J.-C., 2010. Gaussian approximations of fluorescence microscope PSF models. Applied Optics 46 (10), 1819-1829.

Zhou, W., Li, H., Zhou, X., 2008. 3D Dendrite Reconstruction and Spine Identification. IN Medical Image Computing and Computer-Assisted Intervention - MICCAI, 18-26.

Zipfel, W. R., Williams, R. M., Webb, W. W., 2003. Nonlinear magic: multiphoton microscopy in the biosciences. Nature Biotechnology 21 (11), 1369-1377. 


\section{Figures}

\section{A. Data Ceneration}

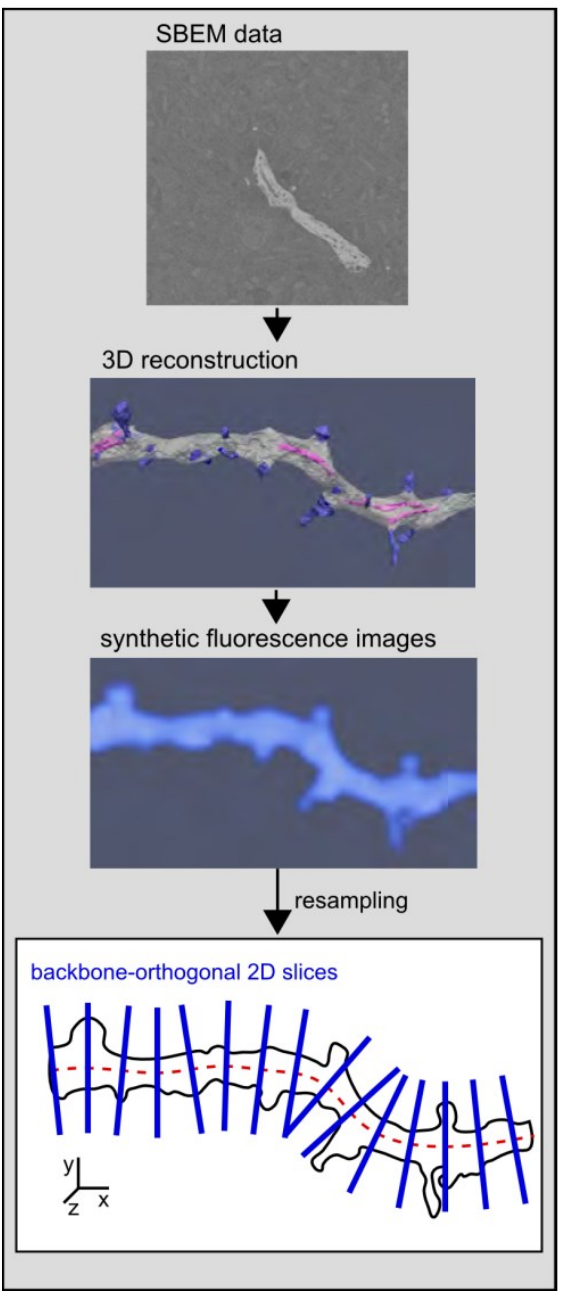

B. Model Computation

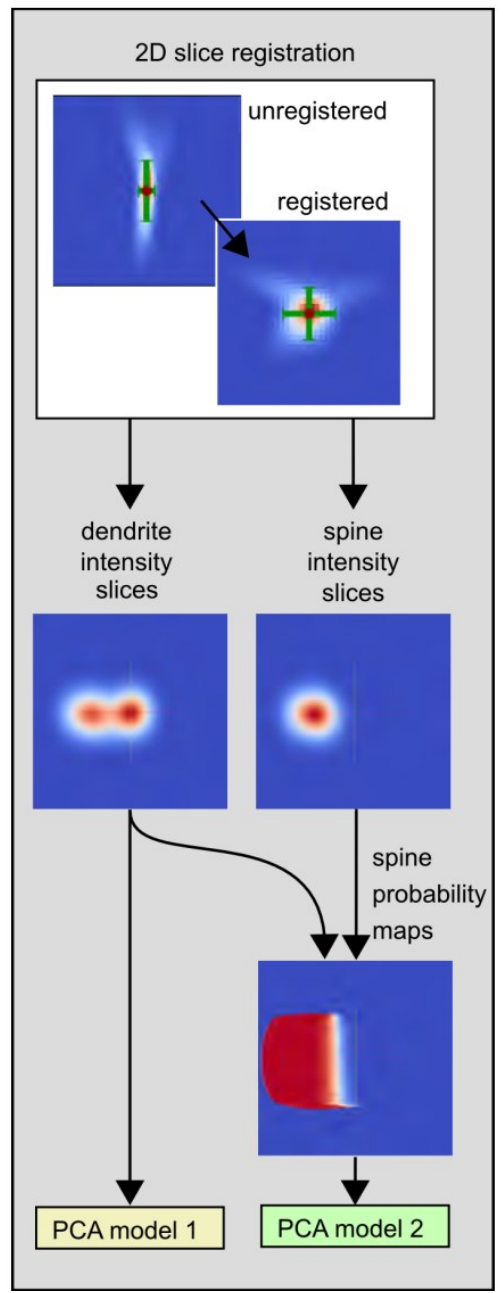

C. Spine Prediction and Segmentation

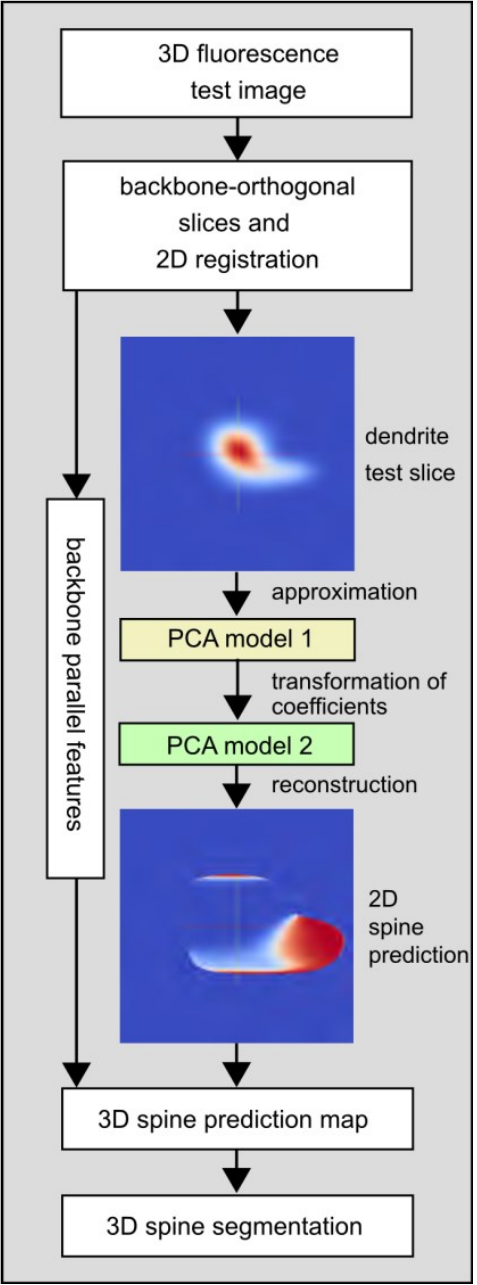

Figure 1: Workflow of model computation and spine prediction. (A) Data generation. From SBEM raw data, a volume reconstruction and synthetic fluorescence images (SFI) are computed. (B) Model computation. From the SFI of dendrites including spines and SFI of spines only, 2D slices are extracted, dendrite/spine intensity maps and spine probability model computed. (C) Spine prediction and segmentation. From a test image, 2D slices are extracted and a spine prediction is computed, using the probability models. Combined with additional backbone-parallel features, a 3D spine prediction map is computed and binarized to achieve 3D spine segmentation. 


\section{A Backbone Parallelism}

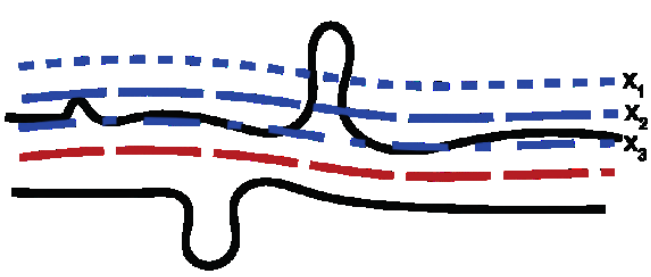

C Backbone Parallel Feature Value

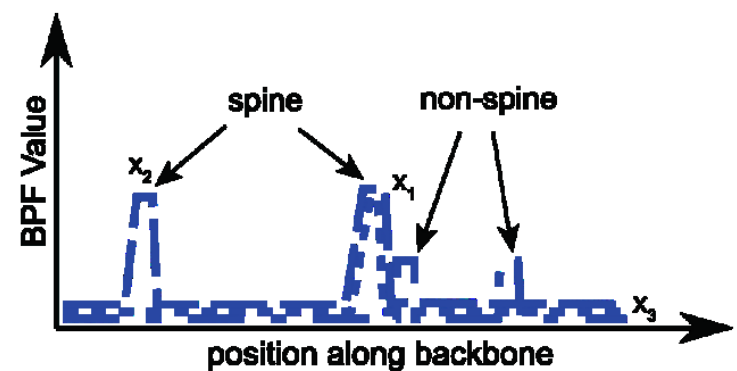

B Dendrite Intensity Profile

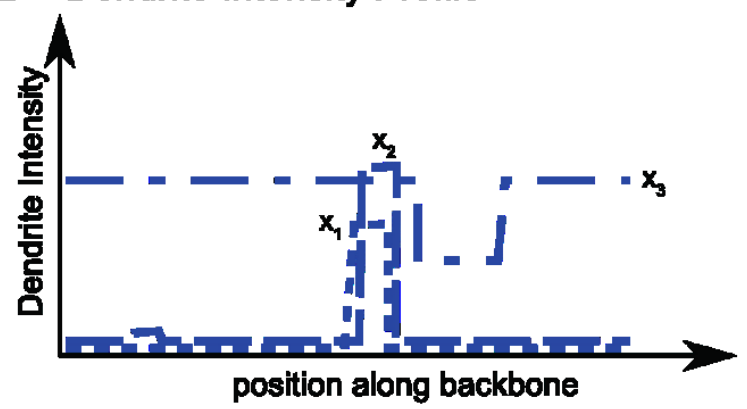

D Intra-Slice Relation

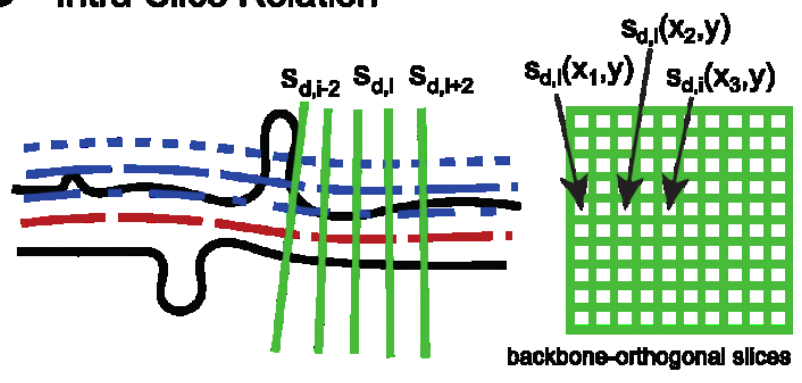

Figure 2: Concept of backbone-parallel features. (A) Dendrite with backbone (red dashed line) and backbone-parallel profiles (blue lines $x_{1}, x_{2}$ and $x_{3}$ ). (B) Dendrite intensity profile through backboneparallel profiles $x_{1}, x_{2}$ and $x_{3}$. (C) Backbone-parallel feature values (schematic) along profiles $x_{1}, x_{2}, x_{3}$. (D) Visualization of relation between profiles $x_{1}$ to $x_{3}$ and backbone-orthogonal slices $s_{d, i}$. The profile values are sampled from the backbone-orthogonal slices $s_{d, i}$ at all pixel positions $x$ and $y$. The right panel shows the location of the profiles $x_{1}, x_{2}$ and $x_{3}$ in the $2 D$ backbone-orthogonal slice $s_{d, i}$. Further slices in a local neighborhood $i-l$ to $i+l$ are used to compute the backbone-parallel feature. 
A
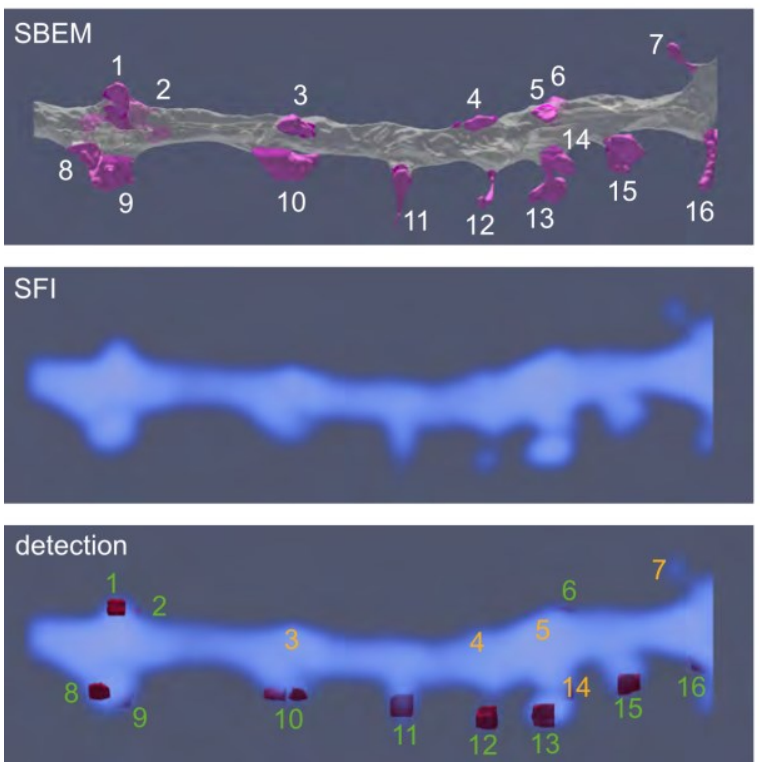

B
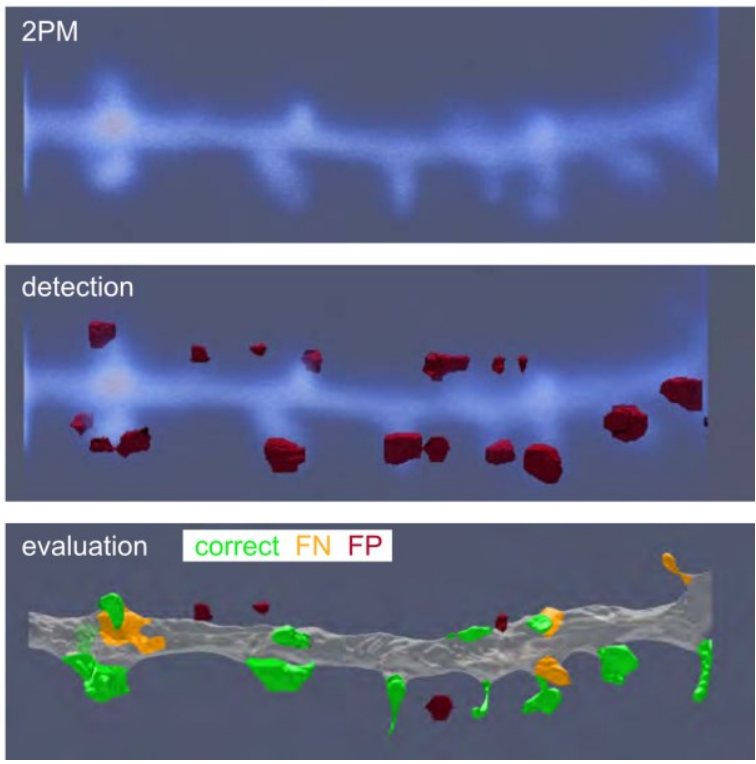

Figure 3: Automated spine detection. (A) EM reconstruction of dendrite \#6 with its 16 spines (top), synthetic fluorescence image (middle), and segmentation result of SFI data (bottom, 11 spines detected). (B) Live cell 2-photon microscopy data (top, dendrite \#6) and segmentation result (middle). True positives (green), false positives (red) and false negatives (orange) are labeled on the correlative EM reconstruction (bottom).

A Orientation of false negatives

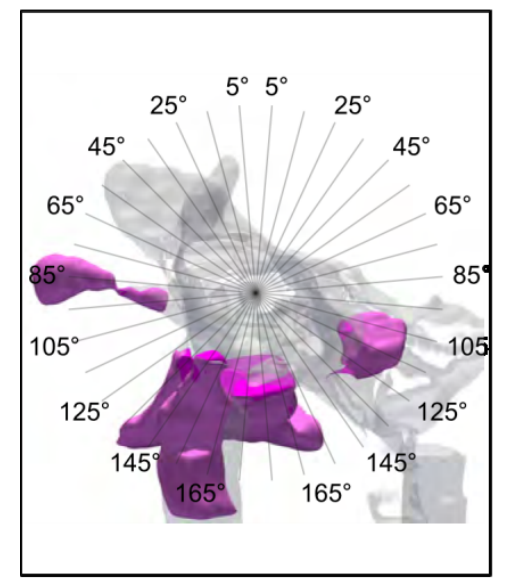

B

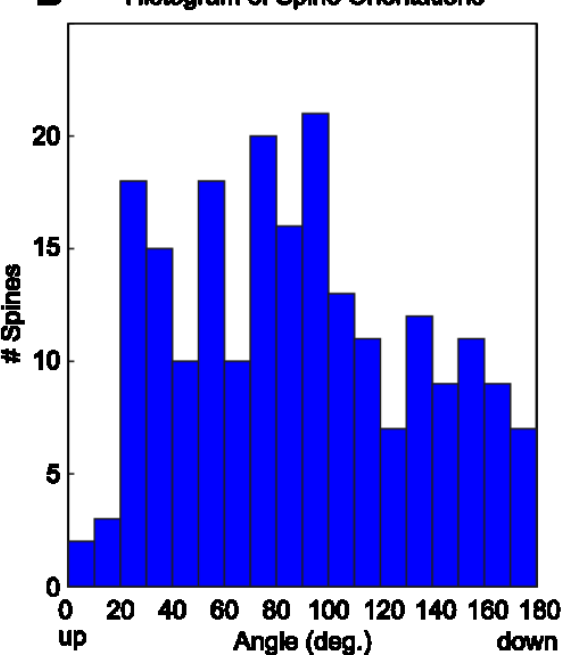

C Spine Orientations, Polar Plot

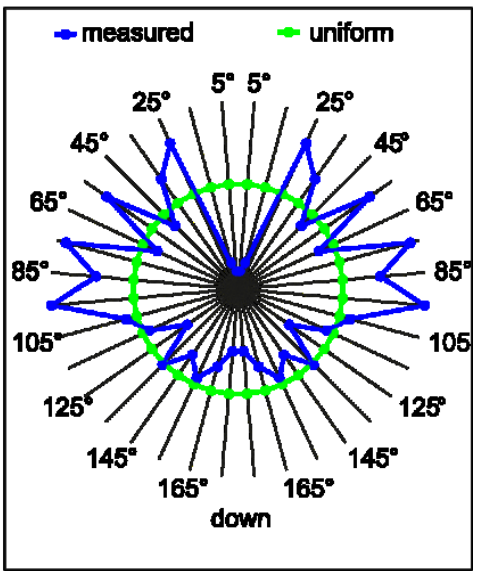

Figure 4: Orientation of spines in organotypic hippocampal cultures. (A) Automatic spine detection in 2PM fluorescence data tends to miss spines that are hidden below the dendrite (Dendrite \#6, pink: false negatives, gray: true positives) due to the strong filtering properties of the PSF in axial direction. (B) Histogram of spine angles based on SBEM data, with $0^{\circ}$ pointing upwards and $180^{\circ}$ pointing downwards ( $n=213$ spines). (C) Polar plot of spine orientation data (blue, mirrored on the vertical axis), compared to a perfectly isotropic distribution (green). Spines protruding upwards from the dendrite are very rare in organotypic slice cultures, while lateral spines are overrepresented. 

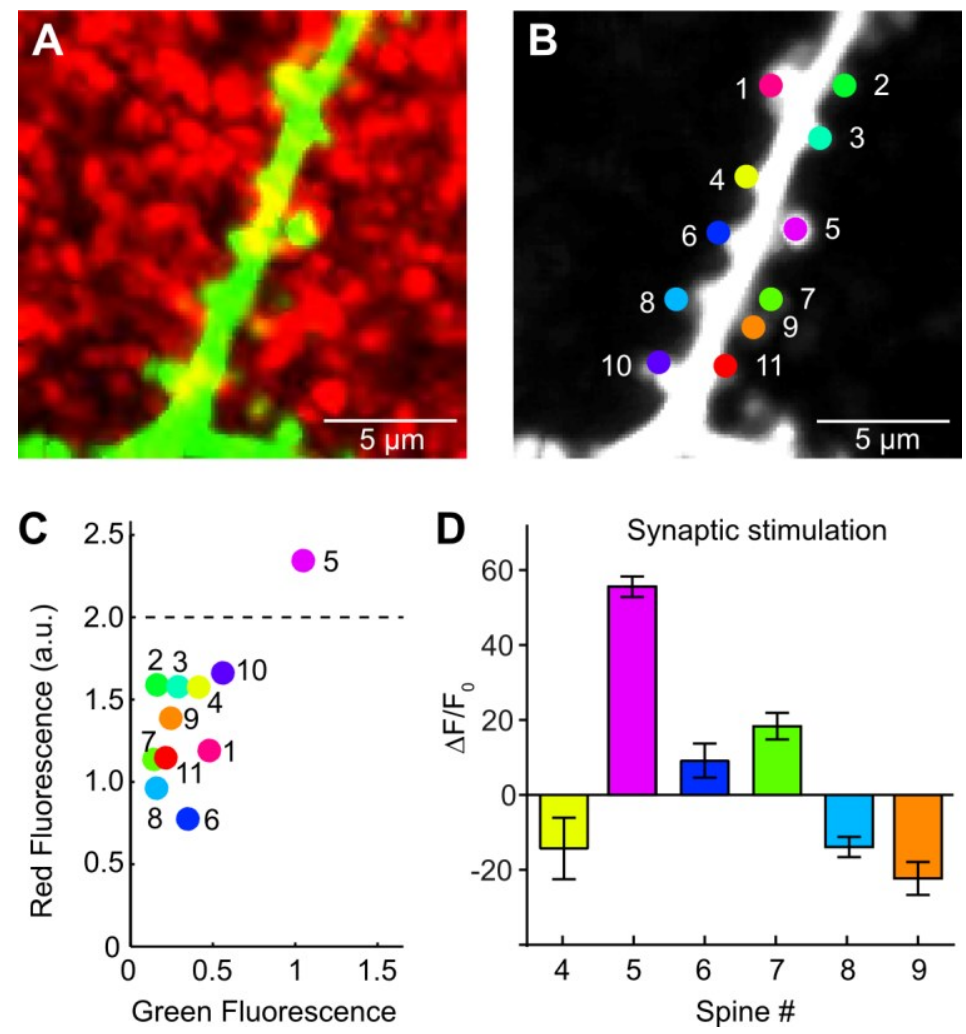

Figure 5: Automatic detection of synaptically connected spines. (A) Two-photon image (MIP) of live organotypic culture. Axons express a light-activated channel and fluorescently labeled vesicle clusters (red). Dendrite express the genetically encoded calcium sensor GCaMP3 and CFP. (B) Automatic spine detection based on CFP fluorescence (volume) of spiny dendrite. (C) Result of automatic two-channel analysis. Spine \#5 displays a particularly high value in the red channel, indicating close proximity to a red fluorescent terminal. (D) Verification of functional synaptic contact by two-photon calcium imaging during optogenetic stimulation. Spine \#5, but no other spine in the analyzed section, displays light-induced calcium transients, indicating the presence of a functional presynaptic terminal. Mean and SEM of 5-10 trials are plotted for every spine. 

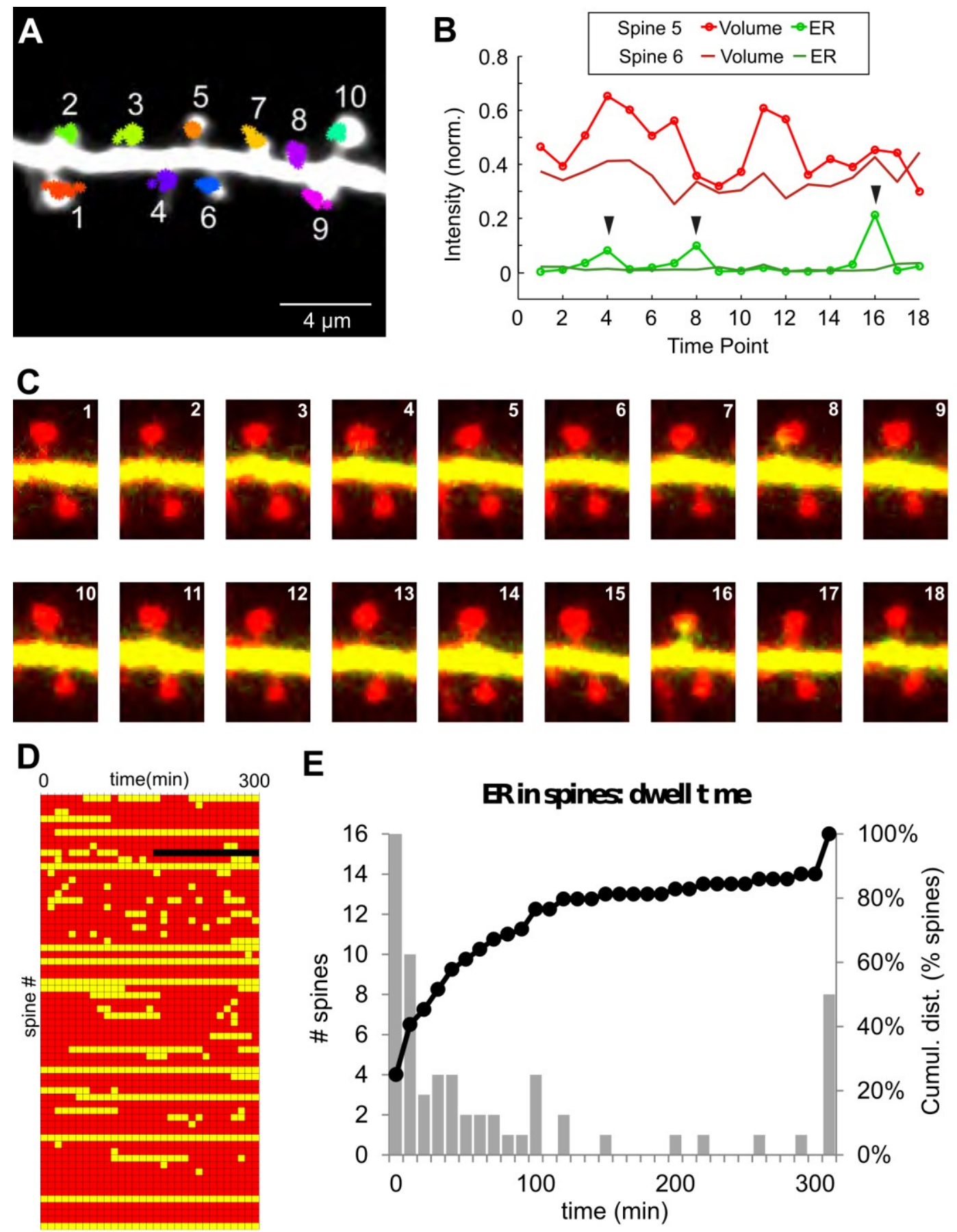

$\mathbf{E}$

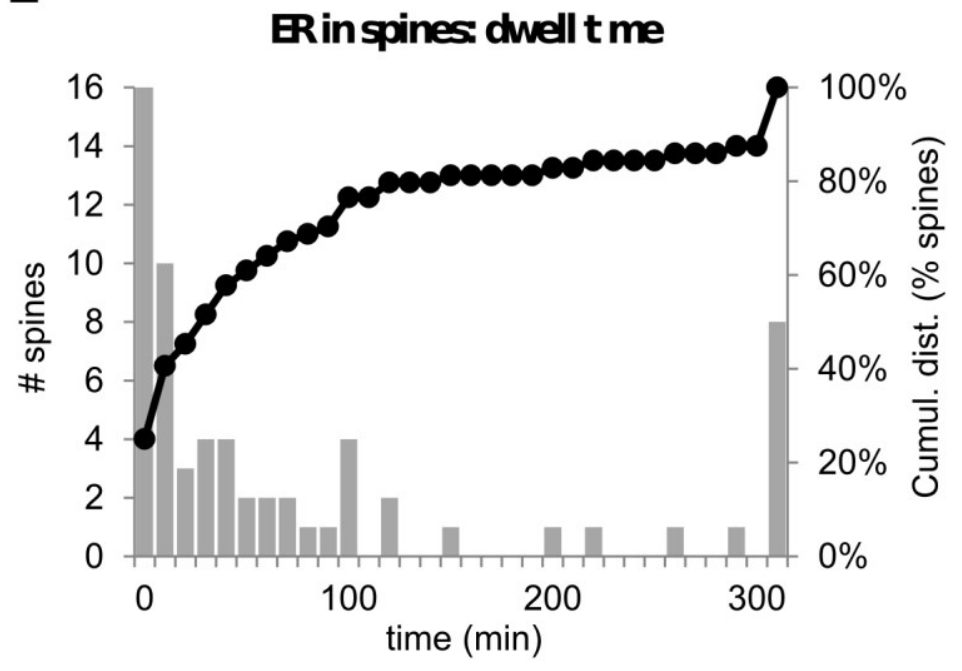

Figure 6: Automated detection of endoplasmic reticulum (ER) invading dendritic spines. (A) Automated spine tracking over time. Stars indicate the automatically detected center of mass of every spine at every time point. Colors indicate automatically assigned spine identities. Background shows MIP of the first time point. (B) Automatically tracked intensities of spine \#5 and \#6 in red fluorescence channel (spine volume, labeled with dsRed) and green fluorescence channel (ER, labeled with GFP). Spine \#5 shows strong volume fluctuations and seems to be invaded by ER at 3 time points (black triangles, green intensity exceeding $2 \sigma$ of baseline). (C) Input images of spine \#5 (pointing up) and spine \#6 (pointing down) over all 18 time points (MIPs). Overlay of red and green channels results in yellow ER signal. In frames 4, 8 and 16, ER invasion into spine \#5 is evident, while spine \#6 is never invaded. (D) Schematic representation of a time-lapse experiment over $300 \mathrm{~min}$, sampling dendritic morphology every $10 \mathrm{~min}$. Spines were detected automatically. Red squares 
represent spines without $E R$, yellow squares spines containing $E R$ ( $n=60$ spines, 1 dendrite). (E) Dwell times of ER in spines are highly variable. Most visits last $<10 \mathrm{~min}$, but the distribution has a long tail towards very long residence times $(>5 h)$. Within $5 h, 88 \%$ of spines were visited by ER at least once. 


\section{Supplementary material}
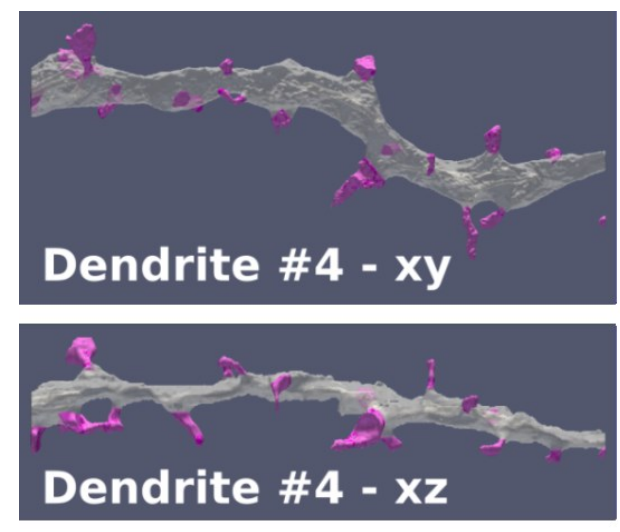
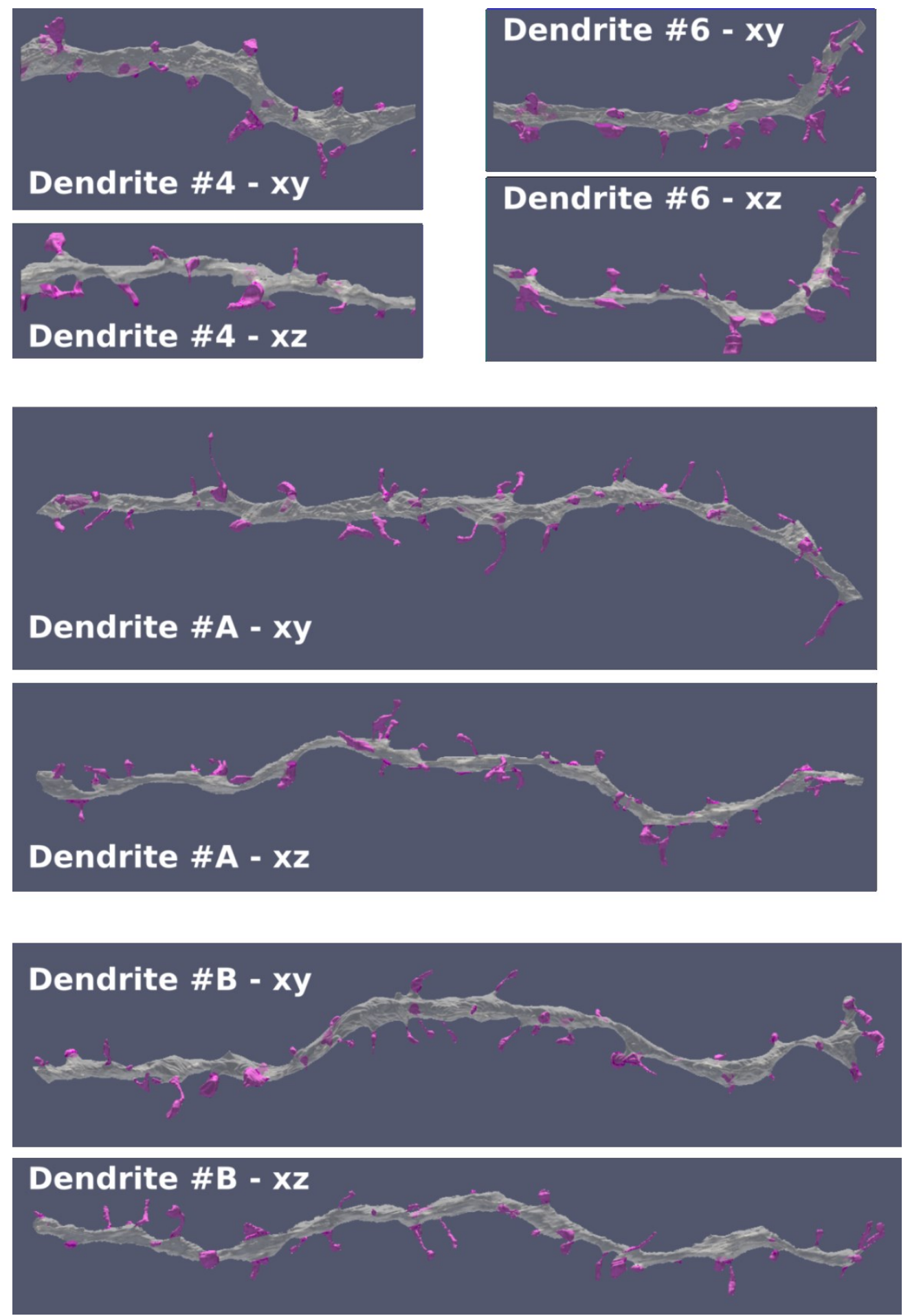

\section{Supplemental Figure S1: Stretches of spiny dendrite used for model computation}

Reconstruction and labeling of spines was done manually on electron microscopy data (SBEM). Enclosed mitochondria were also reconstructed and subtracted from intracellular volume before SFI generation. Dendrite \#4 and \#6 are part of the correlative light/electron microscopy dataset. Data available at www.spinedetection.com 

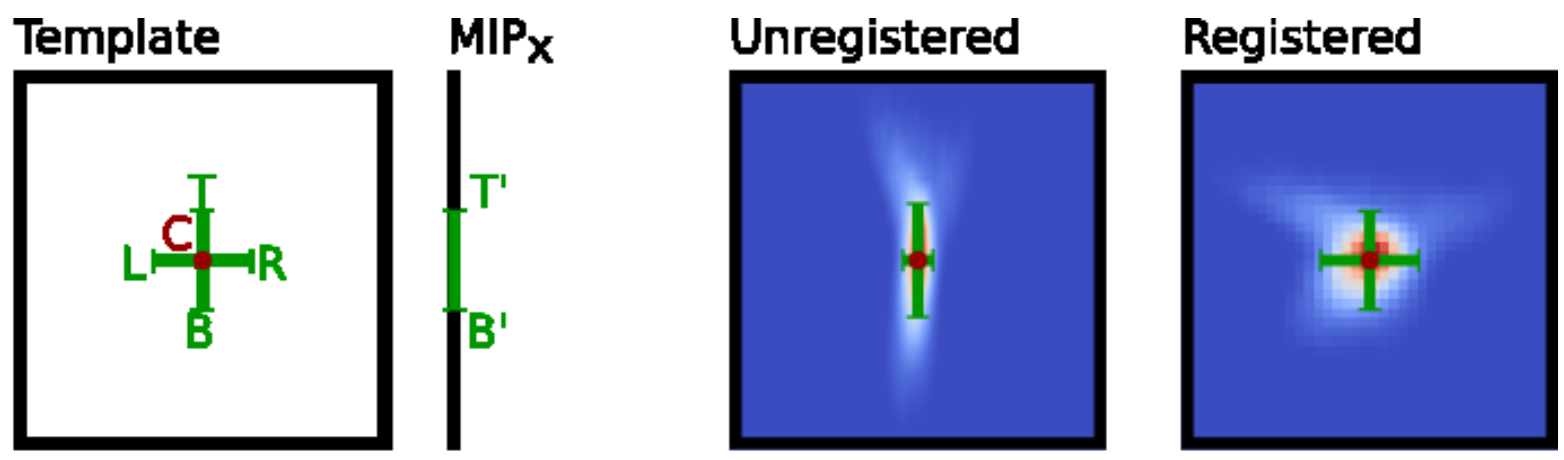

MIPy

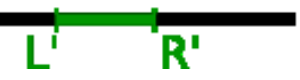

\section{Supplemental Figure S2: Registration of backbone-orthogonal slices}

From the slice center (C) to left (L), right (R), top ( $T$ ) and bottom (B), the distance at which the intensity fell under a given threshold was measured. To minimize the influence of spines, the smaller value from each pair (left/right and top/bottom) was used. In addition, center-to-threshold distance was measured in maximum intensity projections (MIP, 1D) along the $x$ - and $y$-axis $\left(L^{\prime}, R^{\prime}, T^{\prime}, B^{\prime}\right)$. For robust scaling, the average of both measurements (along centerline and in MIP) was used for registration (scaling). 
A
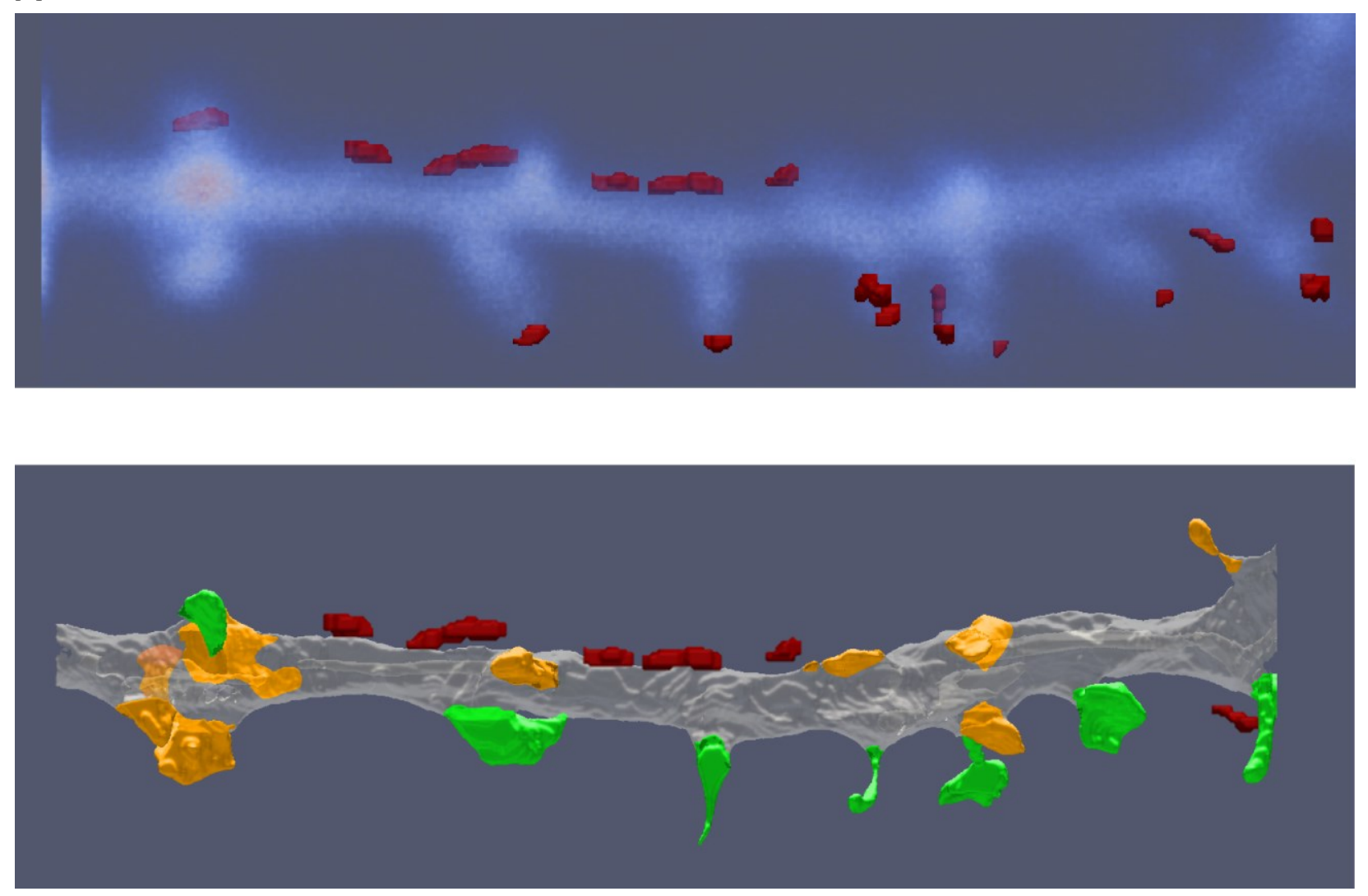

B

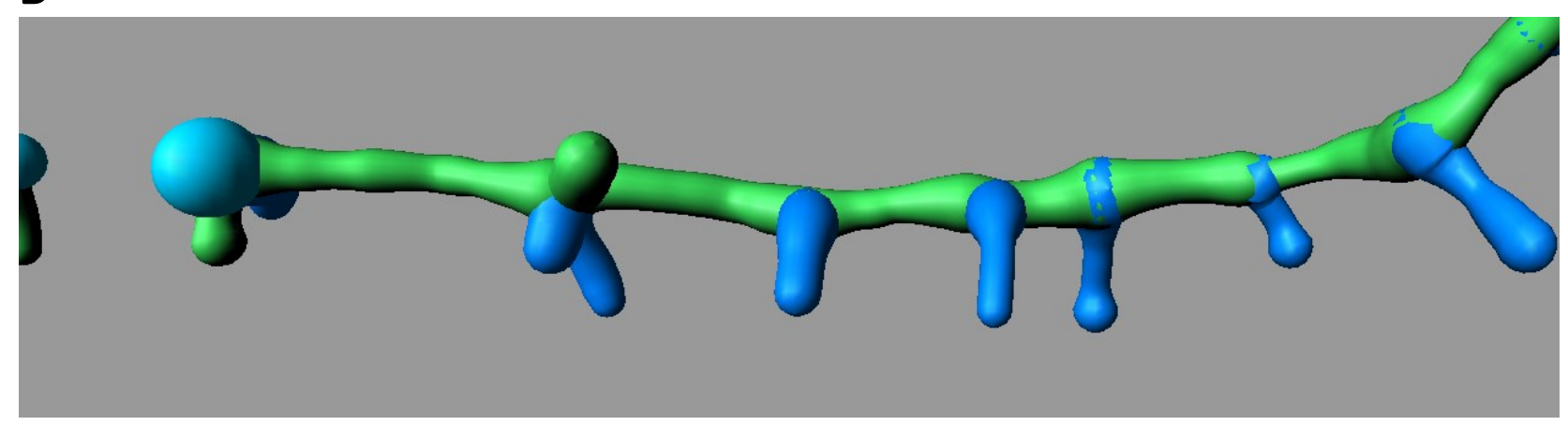

\section{Supplemental Figure S3: Segmentation results with NeuronIQ and Imaris}

A) Segmentation result of NeuronIQ 2.0 on two-photon image of dendrite \#6. Below: Comparison with SBEM data. Green: true positives. Red: false positives. Orange: false negatives. B) Segmentation result of Imaris 7.7 filament tracer (Bitplane AG, Zürich, Switzerland). This program is designed to start backbone reconstruction from the cell body which was outside our images. As the need to find a seed point (large blue sphere) interfered with spine detection in our datasets, we did not score the results numerically. Some spines were classified as side branches of the dendrite (green). 